\title{
Cobertura do citopatológico de colo uterino em um município paranaense: impacto da pandemia Sars-Cov-2
}

\section{Cervical cytopathological coverage of the a paranaense municipality: impact of pandemic Sars-Cov-2}

Marcieli Borba do Nascimento ${ }^{1}$, Tatiane Baratieri², Ellen Cristina Bordelack ${ }^{3}$, Matheus da Cunha Paris ${ }^{4}$

1. ORCID: https://orcid.org/0000-0002-8087-0801. Graduanda em Enfermagem. Universidade Estadual do Centro-Oeste, Guarapuava, Paraná, Brasil. E-mail: marcieli_bn@hotmail.com

2. ORCID: https://orcid.org/0000-0002-8087-0801. Doutora em Saúde Coletiva pela Universidade Federal de Santa Catarina (2020), docente efetiva no curso de Enfermagem da Universidade Estadual do Centro-Oeste, Guarapuava, Paraná, Brasil. E-mail: baratieri.tatiane@gmail.com

3. ORCID: https://orcid.org/0000-0001-5677-4927. Graduanda em Enfermagem. Universidade Estadual do Centro-Oeste, Guarapuava, Paraná, Brasil. E-mail: ellencristinabordelack@gmail.com

4. ORCID: https://orcid.org/0000-0001-5373-1593 Graduando em Enfermagem. Universidade Estadual do Centro-Oeste, Guarapuava, Paraná, Brasil. E-mail: matheusparis91@gmail.com

CONTATO: Autor correspondente: Marcieli Borba do Nascimento. Endereço: R. Simeão Varela de Sá, 153 , kit 04 - Guarapuava - PR. Telefone: (42) 99976-7792. E-mail: Marcieli_bn@hotmail.com

RESUMO A pesquisa buscou analisar a cobertura da prevenção do câncer cérvico-uterino em um município do Paraná. Trata-se de um estudo ecológico, descritivo, retrospectivo, abordagem quantitativa com dados de fonte secundária, entre 2016-2020, ao concernente faixa etária, adequabilidade e amostras alteradas, motivo e periodicidade. Após o levantamento destes dados, as informações foram tabuladas e em tabelas no Excel 2013, por frequência 
absoluta e relativa, cálculo da taxa de cobertura e análise descritiva destes dados. Houve captação de 143.936 citopatológicos de colo uterino em no município no quinquênio analisado, 140.302, estavam na faixa etária de 25-64 anos. A maior taxa de cobertura em rastreamento para a idade preconizada foi observada em 2017 (75\%), com decréscimo em 2020. O município em estudo não conseguiu alcançar indicadores de cobertura do rastreamento de câncer de colo do útero na idade preconizada, sugerindo falhas na captação ou no rastreamento oportuno, necessitando de novas estratégias de rastreamento, bem como aprimoramento das já existentes, como busca ativa, divulgação da oferta de coleta todos os meses do ano, acompanhamento de gestantes e a captação para realização do citopatológico e a vacinação de adolescentes e jovens adultos contra o HPV.

DESCRITORES: SARS-CoV-2. Neoplasias do Colo Uterino. Epidemiologia. Saúde Pública. Saúde da Mulher.

ABSTRACT The research sought to analyze the coverage of cervical cancer prevention in a municipality in Paraná. This is an ecological, descriptive, retrospective, quantitative approach with data from a secondary source, between 2016-2020, concerning age, suitability and changes, reason and frequency. After collecting the data, the information was tabulated and in tables in Excel 2013, by absolute and relative frequency, calculation of the coverage rate and descriptive analysis of this data. There was an uptake of 143,936 cervical cytopathologicals in the city in the five years analyzed, 140,302, were in the age group of 25-64 years. The highest coverage rate in screening for a recommended age was observed in 2017 (75\%), with a decrease in 2020. The municipality under study was unable to achieve coverage indicators for cervical cancer screening at the recommended age, suggesting failures in capturing or timely screening, requiring new screening strategies, as well as improvement of existing ones, such as active search, dissemination the offer of collection every month of the year, monitoring of pregnant women and the recruitment to perform the Pap smear and vaccination of adolescents and young adults against HPV.

DESCRIPTORS: SARS-CoV-2. Uterine Cervical Neoplasms. Epidemiology. Public Health. Women's Health. 


\section{INTRODUÇÃO}

1 undialmente, neoplasias cérvico-uterinas são reconhecidas como o quarto tipo de

neoplasia mais comum entre as mulheres, correspondendo a aproximadamente 530 mil casos novos e 265 mil óbitos por ano. Tendo por característica ser um relevante problema de saúde pública, com denominadores altos especialmente em regiões menos desenvolvidas, locais estes onde, em média, 70,0\% dos casos são diagnosticados ${ }^{1}$.

Tal neoplasia tem origem centrada na infecção persistente por certos tipos de papilomavírus humano (HPV), principalmente os tipos 16 e 18. O vírus frequentemente invade o organismo por meio de infecção genital, através de relação sexual sem preservativo, com forte tendência de permanecer latente no organismo, de modo a não fomentar indícios de sua presença por anos, mas alguns casos passam por modificações celulares que podem evoluir para o câncer ${ }^{2}$. As alterações são descobertas facilmente no exame citopatológico (Papanicolaou) sendo passíveis de tratamento e cura na quase totalidade dos casos quando descobertas inicialmente ${ }^{3}$. De modo amplo, a infecção pelo HPV representa pertinência, visto que, em média $80 \%$, das mulheres sexualmente ativas irão se infectar ao longo da vida. Além disso, aproximadamente 290 milhões de mulheres no mundo são portadoras do HPV'.

Notoriamente, a conclusão diagnóstica para câncer é descrita com desfecho raro, mesmo na presença da infecção pelo HPV, pois a Atenção Básica (AB), busca identificar as lesões em estágios iniciais, evitando sua progressão para casos avançados. Logo, a infecção pelo HPV se faz fator necessário, mas não suficiente, para o desenvolvimento do câncer cervical uterino ${ }^{2,3}$.

Outros fatores que aumentam o risco de desenvolver esse tipo de câncer são: início precoce da atividade sexual (acho interessante colocar a idade se tiver um valor exato); múltiplos parceiros sexuais ao longo da vida; tabagismo (estando diretamente relacionada à quantidade de cigarros fumados), visto que o tabaco apresenta capacidade de agir diretamente nos evolutivos da doença; cita-se também o uso prolongado de pílulas anticoncepcionais ${ }^{4,5,6}$.

A cada ano do triênio 2020-2022, no Brasil, o número de casos novos de câncer do colo do útero é de aproximadamente 16.590, com uma incidência estimada de 15,43 casos a cada 100 mil mulheres ${ }^{1}$.

O cenário pandêmico imposto pelo Sars-Cov-2, reconhecido em março de 2020, vem abalando diferentes setores na sociedade. Uma grande preocupação deste período condiz ao atraso no diagnóstico de doenças crônicas, ou manejo tardio de sua evolução, como os casos de câncer. Nos Estados Unidos da América (EUA), o rastreamento de câncer de mama apresentou queda acima de $90 \%$ no período de $2017-2020^{7}$. Na lite- 
ratura é estimado de que março a maio de 2020, 88.000 casos de câncer deixaram de ser diagnosticados o que levará a graves repercussões no futuro ${ }^{8,9}$.

Neste sentido, o exame citopatológico, método este indubitável, rápido e efetivo na detecção precoce de alterações da cérvice uterina ${ }^{10}$, passou a ser realizado restritamente durante a pandemia. De acordo com uma Nota Técnica emitida pelo Ministério da Saúde, faz-se necessário contrabalançar caso a caso diante do risco de adiamento do rastreamento de câncer e o risco de contágio com o desenvolvimento da COVID-19, os prognósticos podem se associar diretamente a piores desfechos ${ }^{11}$.

Os aspectos positivos em relação à diminuição da incidência do câncer de colo do útero em países que estavam em processo de transição socioeconômica, devido às implementações de programas de prevenção, como a realização do Papanicolau, eram citadas antes da pandemia ${ }^{3}$.

Quando se busca contrabalançar o que é favorável ou não de rastreamento, os benefícios são: prognóstico da doença favorável, com tratamento efetivo e menor impacto no concernente à qualidade de vida e à morbidade. Já os malefícios incluem resultados falso positivos, que geram ansiedade e excesso de procedimentos, resultados falso negativos, levando à falsa tranquilidade do indivíduo, além de sobrediagnóstico e sobretratamento ${ }^{11}$.

Conforme o exposto, é que se justifica a realização deste estudo, visto que o rastreamento de câncer de colo uterino é de extrema importância para a prevenção e diagnóstico precoce e, em outros países já é demonstrada uma queda no rastreamento de alguns tipos de câncer. Assim objetivou analisar o indicador de cobertura da prevenção do câncer cérvico uterino no município de Guarapuava, respondendo a seguinte questão: A pandemia de Sars-Cov-2 vem causando impacto no rastreamento de câncer de colo uterino?

\section{MÉTODO}

Trata-se de um estudo ecológico, descritivo, retrospectivo, com abordagem quantitativa utilizando dados obtidos em fonte secundária, realizado no município de Guarapuava, região centro-sul do Paraná, com informações referentes ao período de 2016 a 2020.

A população estimada para o município de Guarapuava, para o ano de 2019, era de 181.504 habitantes, onde 92.697 eram mulheres que se enquadram de acordo com as diretrizes do Ministério da Saúde para o Rastreamento de câncer cervical, ou seja, na faixa etária entre 25 e 64 anos, conforme o preconizado. Considerando que o impacto de diagnostico em mulheres com menos de 25 anos e mais de 64 anos seria menor, o programa preconiza este intervalo de idade como ideal para rastreio ${ }^{13}$. 
Para a coleta dos dados, foi utilizado o banco de dados do Portal de Informações do Ministério da Saúde - DATASUS, através das informações do Sistema de Informação do Câncer (SISCAN), onde estão contidos dados de domínio público.

A população inicial do estudo constituiu-se do total de mulheres que realizaram o exame citopatológico de colo uterino na secretaria de saúde do município no período de 2016 a 2020. Inicialmente a amostra correspondeu a 143.936 citopatológicos coletados. Após, foram excluídas desta análise, coletas realizadas em mulheres com idade menor que 25 anos (21.568). Por conseguinte, a amostra de estudo correspondeu a 122.368 exames realizados no ultimo quinquênio em Guarapuava.

Para a captação de dados, consideraram-se as variáveis: faixa etária da realização do exame de 25 a 64 anos e 65 anos ou mais; adequabilidade da amostra e amostras com alterações; se foi exame de rastreamento, repetição ou segmento; a periodicidade de realização do exame (se foi feito no mesmo ano, a cada 1 ano, 2 anos, 3 anos, 4 anos ou mais). Após o levantamento destes dados, as informações foram tabuladas e transcritas em tabelas no software Microsoft Office Excel 2013, por frequência absoluta e relativa, e em sequência, procedeu-se à análise descritiva destes dados.

Foi empregado o cálculo de taxa de rastreamento para obter os valores da cobertura do exame no município a cada ano, o referido valor é obtido por meio da divisão: $\mathrm{n}^{\circ}$. Procedimentos realizados em determinado ano/População-alvo (25-64 anos) x 100.

Destarte ainda que, o trabalho respeitou os preceitos éticos de pesquisa que envolve seres humanos, foi tomando como base a Resolução $n^{\circ} 510$, do Conselho Nacional de Saúde, que regulamenta estudos com dados de acesso e domínio público, o estudo dispensou o registro e avaliação pelo Comitê de Ética em Pesquisa (COMEP) não requerendo a utilização de nenhum tipo de identificação da amostra.

\section{RESULTADOS}

Durante o período de 2016 a 2020, foram realizados 143.936 exames citopatológico de colo uterino em mulheres residentes no município de Guarapuava, destas 140.302, apresentaram-se na faixa etária preconizada para rastreio.

Conforme mostra a Tabela 1, em relação a periodicidade de realização do exame em todos os anos analisados, o intervalo de 1 ano prevaleceu com maior proporção em todos os anos, além de ter maiores índices na faixa etária preconizada. 
Tabela 1. Realização do exame citopatológico de colo do útero por ano, faixa etária e periodicidade, no município de Guarapuava - PR, 2016-2020.

\begin{tabular}{|c|c|c|c|}
\hline Ano & Periodicidade & 25 a 64 anos n (\%) & 65 anos ou mais $n(\%)$ \\
\hline \multirow{8}{*}{2016} & Mesmo ano & $221(3,8)$ & $16(3,7)$ \\
\hline & 1 ano & $2.352(40,0)$ & $181(41,6)$ \\
\hline & 2 anos & $1.638(27,9)$ & $108(24,8)$ \\
\hline & 3 anos & $496(8,4)$ & $22(5,1)$ \\
\hline & 4 anos ou mais & $245(4,2)$ & $11(2,5)$ \\
\hline & Mesmo ano & $157(2,9)$ & $13(3,4)$ \\
\hline & 1 ano & $1.910(34,7)$ & $137(35,5)$ \\
\hline & 2 anos & $1.716(31,2)$ & $106(27,5)$ \\
\hline \multirow[t]{5}{*}{2017} & 3 anos & $687(12,5)$ & $35(9,0)$ \\
\hline & 4 anos ou mais & $205(3,7)$ & $9(2,3)$ \\
\hline & Mesmo ano & $186(3,3)$ & $11(2,6)$ \\
\hline & 1 ano & $1.893(33,4)$ & $175(41,0)$ \\
\hline & 2 anos & $1.742(30,7)$ & $96(22,5)$ \\
\hline \multirow[t]{5}{*}{2018} & 3 anos & $687(12,1)$ & $50(11,7)$ \\
\hline & 4 anos ou mais & $291(5,1)$ & $19(4,4)$ \\
\hline & Mesmo ano & $316(3,5)$ & $20(2,4)$ \\
\hline & 1 ano & $2.935(32,2)$ & $306(37,5)$ \\
\hline & 2 anos & $2.440(26,8)$ & $186(22,8)$ \\
\hline \multirow[t]{5}{*}{2019} & 3 anos & $1.124(12,3)$ & $89(10,9)$ \\
\hline & 4 anos ou mais & $698(7,7)$ & $62(7,6)$ \\
\hline & Mesmo ano & $40(1,9)$ & $2(1,5)$ \\
\hline & 1 ano & $512(24,2)$ & $29(22,0)$ \\
\hline & 2 anos & $508(24,0)$ & $32(24,2)$ \\
\hline \multirow[t]{2}{*}{2020} & 3 anos & $262(12,4)$ & $9(6,8)$ \\
\hline & 4 anos ou mais & $167(7,9)$ & $8(6,0)$ \\
\hline
\end{tabular}

Fonte: DATASUS (2021).

Para os motivos da realização do exame, o rastreamento destaca-se com maiores índices, no entanto, o cálculo da taxa de cobertura de citopatológico encontra-se abaixo do recomendado. A maior taxa de cobertura em rastreamento nas mulheres com a idade preconizada foi observada em 2017 (75\%), decrescendo em 2020, alcançando uma cobertura de apenas $20 \%$. Além disso, nota-se uma redução de cerca de $70 \%$ no rastreamento quando comparados os anos de 2019 e 2020. A Tabela 2 ilustra de forma anual a realização de exames coletados, devidamente enquadrados no rastreamento. 
Tabela 2. Distribuição de exames citopatológico de colo do útero realizados por ano, por faixa etária e motivo de indicação de acordo com a taxa de cobertura, em Guarapuava - PR, 2016 - 2020.

\begin{tabular}{lllllll}
\hline Ano & \multicolumn{2}{c}{ Rastreamento } & \multicolumn{2}{c}{ Repetição } & \multicolumn{2}{c}{ Segmento } \\
& $\begin{array}{l}\text { 25-64 anos/ } \\
\text { Taxa de } \\
\text { Cobertura* }\end{array}$ & $\begin{array}{l}\text { Taxa de } \\
\text { Cobertura* }\end{array}$ & $\begin{array}{l}\text { Taxa de } \\
\text { Cobertura* }\end{array}$ & $\begin{array}{l}\text { Taxa de } \\
\text { Cobertura* }\end{array}$ & $\begin{array}{l}\text { Taxa de } \\
\text { Cobertura* }\end{array}$ & $\begin{array}{l}\text { Taxa de } \\
\text { Cobertura* }\end{array}$ \\
\hline $\mathbf{2 0 1 6}$ & $26.530(55,2)$ & $1.618(21,3)$ & $83(0,2)$ & $2(0,0)$ & $115(0,2)$ & $3(0,0)$ \\
$\mathbf{2 0 1 7}$ & $36.461(75,0)$ & $2.358(29,8)$ & $135(0,3)$ & $5(0,1)$ & $157(0,3)$ & $10(0,1)$ \\
$\mathbf{2 0 1 8}$ & $33.597(68,2)$ & $2.267(27,3)$ & $139(0,3)$ & $4(0,0)$ & $165(0,3)$ & $10(0,1)$ \\
$\mathbf{2 0 1 9}$ & $33.522(67,3)$ & $2.019(23,3)$ & $158(0,3)$ & $4(0,0)$ & $165(0,3)$ & $8(0,1)$ \\
$\mathbf{2 0 2 0}$ & $10.192(20,2)$ & $410(4,5)$ & $112(0,2)$ & $6(0,1)$ & $125(0,2)$ & $14(0,2)$ \\
\hline
\end{tabular}

Fonte: DATASUS (2021).

*Taxa em porcentagem.

Conforme nota-se na Tabela 3, a proporção dos exames citopatológicos alterados na faixa etária preconizada para o rastreamento se manteve na casa dos $20 \%$, com diminuição apenas em 2017 e 2018 que foi cerca de 15\%. Porém, há um menor número de exames realizados quando comparado a anos anteriores, o que significa que muitas pessoas com alterações não realizaram o rastreamento.

Tabela 3. Realização do exame citopatológico de colo do útero de acordo com ano e número de alterações identificadas no município de Guarapuava - PR, 2016-2020.

\begin{tabular}{lllll}
\hline Ano & Idade & Alterados $\mathbf{n}(\%)$ & \multicolumn{1}{c}{ Idade } & Alterados $\mathbf{n}(\%)$ \\
& $25-64$ anos & $25-64$ anos & $>65$ anos & $>65$ anos \\
\hline $\mathbf{2 0 1 6}$ & 26.530 & $5.879(22,1)$ & 1.622 & $435(27,0)$ \\
$\mathbf{2 0 1 7}$ & 36.461 & $5.508(15,1)$ & 2.369 & $386(16,2)$ \\
$\mathbf{2 0 1 8}$ & 33.597 & $5.675(15,5)$ & 2.275 & $427(19,0)$ \\
$\mathbf{2 0 1 9}$ & 33.522 & $9.115(27,1)$ & 2.029 & $817(40,2)$ \\
$\mathbf{2 0 2 0}$ & 10.192 & $2.116(21,0)$ & 429 & $131(30,5)$ \\
\hline
\end{tabular}

Fonte: DATASUS (2021).

\section{DISCUSSÃO}

A faixa etária preconizada pelas organizações de saúde penhoram que se submetam ao rastreamento de alterações cérvico-uterinas mulheres assintomáticas, o que corresponde a aproximadamente $33,3 \%$ da população feminina na faixa etária de 25 a 64 anos no país ${ }^{3}$. Para o Brasil, as maiores coberturas foram observadas entre mulheres de 35 a 54 anos $^{12}$. 
Os dados deste estudo apontam que, em relação a faixa etária e o segmento no programa de rastreamento, evidentemente as mulheres têm procurado o serviço para realizar o procedimento, mas supostamente indica que o protocolo de rastreio não está sendo aplicado corretamente, visto que de acordo com as diretrizes ministeriais o recomendado é de coleta trienal após duas anuais inalteradas ${ }^{3}$.

No município de Guarapuava/PR, nos últimos anos, percebe-se com maior vigor o rastreamento de forma oportunística, isso é, ao procurar o serviço de saúde por outras causas, as mulheres são captadas para realização do exame Papanicolaou ${ }^{14}$.

A Secretaria Estadual de Saúde do Paraná, preconiza que até 2023 ocorra monitoramento e intensificação de coleta de citologia do colo do útero na população feminina dentro da faixa etária preconizada para rastreamento, melhorando assim o indicador "Razão entre exames citopatológico do colo do útero na faixa etária de 25 a 64 anos e a população feminina na mesma faixa etária"15.

Apesar das recomendações do MS e da meta do Estado do Paraná, o estudo revelou que o município enfrenta dificuldades em relação a coleta fora da faixa etária recomendada, pois muitas pessoas com 65 anos ou mais continuam realizando o exame, o que sugere gastos desnecessários dos serviços públicos.

Vigora no Estado a Linha Guia "Mãe Paranaense", a qual organiza a atenção materno-infantil, no âmbito das ações de pré-natal, parto, puerpério, crescimento e desenvolvimento das crianças até um ano de vida, com objetivo primordial reduzir a mortalidade materna e infantil. No decorrer da gestação vários são os exames realizados a gestante, dentre eles o citopatológico de colo uterino ainda no primeiro trimestre gestacional. A atenção dada as mulheres durante o período gravídico-puerperal facilitam a entrada das mulheres na Rede de Atenção à Saúde e aos cuidados relacionados a doenças, tais como o câncer cervical, no entanto nos leva a pensar nas demais mulheres que por vezes não procuram o serviço de saúde com outras queixas e deixam de ser rastreadas ${ }^{16}$.

Aproximadamente, $20 \%$ a $25 \%$ das coletas têm sido realizadas fora do grupo etário recomendado e aproximadamente $50 \%$ delas com intervalo de um ano ou menos, quando o recomendado são três anos, após dois exames anuais com resultados inalterados, corroborando com os dados encontrados neste estudo ${ }^{13}$.

Estudo conduzido no Acre, considera que a menor realização do exame em mulheres acima de 65 anos é contundente ao fim da idade fértil da mulher, que consequentemente acarretaria em diminuição na busca pelos serviços de saúde, mas infelizmente segundo os autores este é período no qual a gravidade das neoplasias se torna alta ${ }^{17}$. E como pode se notar neste estudo, a faixa etária de 65 anos ou mais foi a que mais apresentou alterações no exame, chegando a 40\% em 2019. 
O padrão mais alimentado de rastreamento no Brasil é o oportunístico, pois as mulheres realizam o Papanicolaou, ao procurarem os serviços de saúde por diversas outras razões ${ }^{13}$. Quando comparado aos programas de rastreamento organizado, em que existe maior sistematização das ações e controle sobre as informações, o modelo oportunístico costuma ser menos efetivo no impacto sobre a morbimortalidade atribuída à condição rastreada, além de ser mais oneroso para o sistema de saúde ${ }^{13}$.

O desenvolvimento do câncer de colo uterino em mulheres até 24 anos é relativamente baixo e, por isso, o rastreamento nesta faixa etária não é considerado eficaz para a deteç̧ão. Além disso, o tratamento de lesões precursoras em adolescentes e mulheres jovens eleva o número de colposcopia, aumentando, consequentemente, a morbidade obstétrica e neonatal em futuras gestações, considerando que a maioria das mulheres nesta idade não possui sua prole definida ${ }^{18}$.

Atualmente o Ministério da Saúde, preconiza a vacina quadrivalente do HPV, para meninas com idade de 9 a 14 anos e meninos entre 11 a 14 anos com esquema de duas doses ao ano, sendo a segunda seis meses após a primeira ${ }^{19}$, assim o município de Guarapuava, segue este esquema de vacinação, o intuito é que ao vacinar os adolescentes e tendo no imunobiológico dois dos tipos principais do vírus envolvidos nas lesões cancerígenas, venha futuramente a reduzi-lo como fator de risco para o desenvolvimento da doença. Entretanto, estudo aponta que a cobertura vacinal, especialmente para a segunda dose do imunobiológico, está aquém do esperado em todo Brasil, o que irá impactar negativamente nos benefícios advindos dessa estratégia ${ }^{20}$.

No Brasil, foi preconizado que até 2022 a cobertura esperada fosse de, no mínimo, $80 \%$ da população-alvo, isso para obtenção de impacto epidemiológico positivo, ou seja, reduzir em torno de 60 a $90 \%$ da incidência do câncer de colo uterino no país ${ }^{21}$. Porém, com a pandemia, o distanciamento social, o medo das pessoas saírem de casa e as restrições dos serviços de saúde, nota-se uma redução da cobertura conforme evidenciado por este estudo e por estudo internacional ${ }^{22}$.

O sucesso de um programa de rastreamento não se dá apenas com o acesso ao primeiro exame, mas sim com o seguimento completo de pessoas que, nesse exame de rastreamento, tenham apresentado resultados alterados. A coleta inadequada do material para análise, o atraso entre o recebimento do resultado do exame, a confirmação diagnóstica e o tratamento adequado podem influenciar na evolução clínica dos casos, implicando aumento de incidência, mas não tendo impacto sobre a mortalidade ${ }^{12}$.

Para que o exame seja eficaz e apto a detectar lesões cancerosas precursoras, é imprescindível coleta adequada do material que será avaliado, tendo como necessários os seguintes fatores: número mínimo de células escamosas e ausência de elementos que prejudiquem a leitura do material, a exemplo: presença de sangue, biócitos ${ }^{23,24}$. 
Para ser considerado um esfregaço satisfatório para avaliação oncótica, é fundamental a presença de células em quantidade representativa, bem distribuídas, fixadas e coradas, cuja visualização permita uma conclusão diagnóstica ${ }^{24}$.

A dificuldade no alcance da meta preconizada neste estudo durante todo o quinquênio (2016-2020) e principalmente em 2020 por conta da pandemia, sugere falhas na captação das mulheres e, possivelmente, a influência da participação da saúde suplementar na realização dos exames. No entanto o município de Guarapuava/PR vem fortalecendo suas ações, o mês de outubro é visto como o mês em que as ações são voltadas a saúde ginecológica, tendo em todo o território brasileiro atividades com foco no rastreamento do câncer de colo do útero e de mama. Recentemente o município contou com apoio de docentes e discentes de um curso de nível superior, que em conjunto com a secretaria de saúde realizaram uma campanha de exames preventivos de câncer de colo de útero em mulheres da localidade, onde a ideia é oportunizar coletas em massa não somente no mês de outubro, mas no decorrer de todo o ano ${ }^{25}$.

No entanto, se faz mensurável dizer que, a priorização de uma faixa etária não significa a impossibilidade da oferta do rastreio às mulheres mais jovens ou mais velhas, mediante assistência de qualidade, uma anamnese bem realizada, escuta ativa para o reconhecimento dos fatores de risco envolvidos e do histórico assistencial da mulher, os quais são impreteríveis para indicação do exame de rastreamento ${ }^{11}$.

Para o município analisado, o maior número de citopatológicos cérvicos-uterinos, ocorreu em 2017 (25\%), seguido por 2018 (24\%). Ao passo que analisarmos exames alterados a cada ano, 2020 representa menores dígitos. A queda de rastreamento, quando comparados os anos de 2019 e 2020, foi de cerca de 70\%, assim como demonstra o estudo realizado nos Estados Unidos da América que avaliou os efeitos da pandemia na redução dos exames de rastreamento do câncer de colo do útero e no diagnóstico do câncer, visto que as unidades de saúde limitaram o acesso, priorizando atendimentos de urgência e emergência e as pessoas saíram menos de casa, além de irem menos aos serviços de saúde por medo de serem infectadas pelo novo coronavírus ${ }^{22}$.

Isso se explica devido ao reconhecimento em 11 de março de 2020, da pandemia da Sars-Cov-2, a qual comprometeu inúmeras facetas da sociedade, ao que condiz com o diagnóstico e o tratamento oncológico neste momento, o foco primordial vem sendo limitar os riscos causados pela infecção pelo vírus Sars-CoV-2 não sendo prejudicial às necessidades de cada paciente, é consenso que a pandemia trouxe e ainda caminha lado a lado com os impactos que a detecção da doença em estágios mais avançados podem causar no futuro, diante do risco de evolução da neoplasia e um pior prognóstico ${ }^{23}$.

Além disso, foram suspensos os procedimentos eletivos, incluindo o rastreamento de câncer, na maioria dos países, diante da necessidade de priorização das urgências e 
redução do risco de disseminação do novo coronavírus (SARS-CoV-2) nos serviços de saúde ${ }^{26}$. Os resultados disso no futuro podem ser agravamentos das morbidades, incremento da mortalidade e filas ou listas de espera maiores ${ }^{27}$, não somente no município em estudo, mas mundialmente verificados.

\section{CONCLUSÃO}

Como pode se notar, o município em estudo não conseguiu alcançar os indicadores de cobertura do rastreamento de câncer de colo do útero na faixa etária preconizada, o que sugere falhas na captação de mulheres e do sistema de rastreamento oportunístico, sugerindo novas estratégias de rastreamento, como é o caso do rastreamento sistematizado.

Apesar de o estudo ter sido realizado apenas na cidade de Guarapuava, os resultados corroboram com os de estudos realizados no exterior, que demonstram uma queda na cobertura do rastreamento por conta da pandemia, o que pode implicar no aumento de morbidades mais complexas e, até mesmo, em um aumento da mortalidade por câncer de colo do útero.

Além disso, este estudo também contribui para os gestores pensarem em formas de captar estas mulheres durante a pandemia, oferecendo um espaço com segurança, fornecendo os EPI's aos profissionais que realizam a coleta e a paciente que realizará o exame, visto que o contato do profissional não será próximo às vias aéreas, então, com o uso adequado de EPI's, controle de contatos de pacientes com COVID, ambiente grande e, se possível, ventilado, as chances de transmissão do coronavírus fica diminuída.

Sugere-se, também, a realização de mais estudos, englobando mais cidades ou estados para analisar a cobertura nacional, bem como estudos que visem criar estratégias para o aumento da cobertura de rastreamento.

\section{REFERÊNCIAS}

1. Brasil. Ministério da Saúde. Instituto Nacional do Câncer José Alencar Gomes da Silva. Estimativa 2020: incidência de câncer no Brasil / Instituto Nacional de Câncer José Alencar Gomes da Silva. - Rio de Janeiro: INCA, 2019 [acesso em: 13 abr 2021]; Disponível em: https://www.inca.gov.br/sites/ufu.sti.inca.local/ files/media/document/estimativa-2020-incidencia-de-cancer-no-brasil.pdf

2. International Agency of Research on Cancer. Working Group on the Evaluation of Carcinogenic Risks to Humans. Human papillomaviruses. Lyon: WHO; IARC, 2007. 636p. (IARC Monographs on the Evaluation of Carcinogenic Risks to Humans, v. 90). Disponível em: https://monographs.iarc.who.int/wp-content/ uploads/2018/06/mono90.pdf

3. Brasil. Ministério da Saúde. Instituto Nacional do Câncer José Alencar Gomes da Silva. Controle do Câncer de Colo do Útero: Fatores de Risco. 2020 [acesso em: 24 abr 2021]; Disponível em: https://www. inca.gov.br/controle-do-cancer-do-colo-do-utero/fatores-de-risco

4. International Agency of Research on Cancer. Working Group on the Evaluation of Carcinogenic Risks to Humans. Human papillomaviruses. Lyon: WHO; IARC, 2007. 636p. (IARC Monographs on the Evaluation of Carcinogenic Risks to Humans, v. 90); [Acesso em: 28 abr 2021]; Disponível em: https://monographs. iarc.who.int/wp-content/uploads/2018/06/mono90.pdf 
5. Torres-Poveda K, Ruiz-Fraga I, Madrid-Marina V, Chavez M, Richardson V. High Risk HPV Infection Prevalence and Associated Cofactors: A Population-Based Study in Female ISSSTE Beneficiaries Attending the HPV Screening and Early Detection of Cervical Cancer Program. BMC Cancer. 2019 dez, p. 1205 [acesso em: 28 abr 2021]; 19(1). doi: https://doi.org/10.1186/s12885-019-6388-4

6. Guedes DHS, Fiorin BH, Santos MVF, Viana KCC, Portugal FB, Silva RA. Factors associated to the human papillomavirus in women with cervical cancer. Rev Rene. 2020 [acesso em:13 mai 2021]. Disponível em: https://doi.org/10.15253/2175-6783.20202143681

7. Ziedan E, Simon KI, Wing C. Effects of state COVID-19 closure policy on non-COVID-19 health care utilization. Cambridge: National Bureau of Economic Research; 2020 [acesso em 28 abr 2021]. Disponível em: https://www.nber.org/system/files/working_papers/w27621/w27621.pdf

8. Komodo Health. Routine chronic disease screenings and oncology biomarker tests plummet during COVID-19. 2020 [acesso em: 27 abr 2021]. Disponível em: https://knowledge.komodohealth.com/hubfs/ white-papers/research-briefs/Komodohealth-covid19-2020-04-28.pdf

9. Maluf F. Os impactos do adiamento de tratamentos do dia a dia e retomada dos procedimentos no setor hospitalar. Panorama ANAPH; 2020 [acesso em: 28 abr 2021]. Disponível em: https://www.anahp.com.br/ pdf/revista-panorama-edicao-76.pdf

10. Dias MFM. Impacto do Programa Nacional de Melhoria do Acesso e da Qualidade nas Ações De Rastreamento Do Câncer De Colo Uterino em Alagoas. Pernambuco. Dissertação [Mestrado em Gestão e Economia da Saúde]. Universidade Federal de Pernambuco; 2016. Disponível em: https://repositorio.ufpe. br/handle/123456789/18549

11. Ministério da Saúde. Instituto Nacional de Câncer José Alencar Gomes da Silva. Nota Técnica - DIDEPRE/CONPREV/INCA - 30/3/2020 Detecção precoce de câncer durante a pandemia de Covid-19. Disponível em: https://www.inca.gov.br/sites/ufu.sti.inca.local/files/media/document/nota-tecnica-deteccao-precoce.pdf

12. Oliveira MM, Andrade SSCA, Oliveira PPV, Silva MMA, Malta DC. Cobertura de exame Papanicolaou em mulheres de 25 a 64 anos, segundo a Pesquisa Nacional de Saúde e o Sistema de Vigilância de Fatores de Risco e Proteção para Doenças Crônicas por Inquérito Telefônico, 2013. Revista Brasileira de Epidemiologia, vol. 21 Supl. 22018 [acesso em: 29 abr 2021]. doi: https://doi.org/10.1590/1980549720180014.supl.2

13. Brasil. Ministério da Saúde. Instituto Nacional de Câncer José Alencar Gomes da Silva. Coordenação de Prevenção e Vigilância. Divisão de Detecção Precoce e Apoio à Organização de Rede. Diretrizes brasileiras para o rastreamento do câncer do colo do útero / Instituto Nacional de Câncer José Alencar Gomes da Silva. Coordenação de Prevenção e Vigilância. Divisão de Detecção Precoce e Apoio à Organização de Rede. - 2. ed. rev. atual. - Rio de Janeiro: INCA, 2016 [Acesso em: 13 abr 2021]; Disponível em: https:// www.inca.gov.br/publicacoes/livros/diretrizes-brasileiras-para-o-rastreamento-do-cancer-do-colo-do-utero

14. Brasil. Ministério da Saúde. Rastreamento. Brasília: Ministério da Saúde, 2010. (Série A. Normas e Manuais Técnicos) (Cadernos de Atenção Primária n. 29). [Acesso em: 01 out. 2021]. Disponível em: https:// aps.saude.gov.br/biblioteca/visualizar/MTlwNg==

15. Paraná. Secretaria de Estado da Saúde do Paraná. Plano Estadual de Saúde Paraná 2020-2023 Curitiba: SESA, 2020. 210 p. [Acesso em: 01 out. 2021]. Disponível em: https://www.conass.org.br/wp-content/uploads/2021/04/PLANO-ESTADUAL-DE-SAU\%CC\%81DE-DO-PARANA\%CC\%81-2020-2023.pdf

16. Paraná. Secretaria de Estado da Saúde do Paraná . Linha Guia Mãe Paranaense. Curitiba: SESA, 2018. 63 p. [Acesso em: 01 out. 2021. Disponível em: https://www.saude.pr.gov.br/sites/default/arquivos_ restritos/files/documento/2020-09/LinhaGuiaMaeParanaense_2018.pdf

17. Borges, MFSO, Dotto LMG, Koifman RJ, Cunha MA, Torres MP. Prevalência do exame preventivo de câncer do colo do útero em Rio Branco, Acre, Brasil, e fatores associados à não-realização do exame. Cadernos de Saúde Pública, vol. 28, no 6, junho de 2012, p. 1156-66 [Acesso em: 29 abr 2021]. doi: https:// doi.org/10.1590/S0102-311X2012000600014

18. Brasil. Ministério da Saúde. Secretaria de Atenção à Saúde. Departamento de Atenção Básica. Controle dos cânceres do colo do útero e da mama. 2. ed. - Brasília: Editora do Ministério da Saúde. (Cadernos 
de Atenção Básica, n 13). 2013:128p. [acesso em: 04 mai 2021]; Disponível em: http://189.28.128.100/ dab/docs/portaldab/publicacoes/cab13.pdf

19. Brasil. Ministério da Saúde. Saúde amplia vacinação contra HPV para mulheres imunossuprimidas com até 45 anos. [Acesso em: 01 out. 2021]. Disponível em: https://www.gov.br/saude/pt-br/assuntos/noticias/saude-amplia-vacinacao-contra-hpv-para-mulheres-imunossuprimidas-com-ate-45-anos

20. Moura, L. L.; Codeço, C. T.; Luz, P. M. Cobertura da vacina papilomavírus humano (HPV) no Brasil: heterogeneidade espacial e entre coortes etárias. [acesso em: 01 out, 2021]. doi: https://doi.org/10.1590/1980549720210001

21. World Health Organization. Cancer Control. Knowledge into ation. WHO guide for efective pogrammes. Switzerland: WHO, 2007 [ Acesso em: 04 de mai 2021]. Disponível em: https://apps.who.int/iris/bitstream/ handle/10665/43743/9241547338_eng.pdf;jsessionid=780140F826D6582ACC42BBC55A84495A?sequence $=1$

22.Bakouny Z, Paciotti M, Schmidt AL, Lipsitz SR, Choueiri TK, Trinh QD. Cancer screening tests and cancer diagnoses during the Covid-19 pandemic. JAMA Oncol. 2021 [acesso em: 08 maios 2021]. Disponível em: https://pubmed.ncbi.nlm.nih.gov/33443549/

23. Geremia, DS, Derner T, Rosa M, Antunes TR, Pitilin EB, Gasparin VA. Avaliação da adequabilidade da coleta do exame citopatológico na Estratégia Saúde da Família. Revista Acreditação: ACRED. 2016 [Acesso em: 29 abr 2021]; 6(11). Disponível em: https://dialnet.unirioja.es/servlet/articulo?codigo=5602111

24. Ceolin R, Nasi C, Coelho DF, Paz AA, Lacchini AJB. Análise do rastreamento do câncer do colo do útero de um município do sul do Brasil. Rev Fun Care Online. 2020 [acesso em: 01 maio 2021]12:406-412. doi: https://doi.org/10.9789/2175-5361.rpcfo.v12.8342

25. Paraná. Agencia de Notícias do Paraná. Alunos da Unicentro fazem mutirão para atender mulheres com exame preventivo de câncer. [Acesso em: 01 out. 2021]. Disponível em: https://www.aen.pr.gov.br/ modules/noticias/article. php?storyid $=115476$

26. Paho - Pan American Health Organization. OMS afirma que COVID-19 é agora caracterizada como pandemia - OPAS/OMS | Organização Pan-Americana da Saúde. 2020 [Acesso em: 14 mai 2021]; Disponível em: https://www.paho.org/pt/news/11-3-2020-who-characterizes-covid-19-pandemic

27. Berlin G, Bueno D, Gribler K, Schulz J. Cutting through the COVID-19 surgical backlog. McKinsey \& Co. 2020 [Acesso em: 14 maio 2021]. Disponível em: https://www.mckinsey.com/industries/healthcare-systems-and-services/our-insights/cutting-through-the-covid-19-surgical-backlog

RECEBIDO: 18/05/2021

ACEITO: 11/10/2021 